without assistance: yet we ignore these facts because we hear bronchial sounds or distant murmur, fail to find dulness in the lower chest zone, and the intercostal spaces do not bulge.

DISCESSION.

Dr. E. Fletcher Ingals of Chicago-The paper which has just been read has many points of interest to many physicians and I think they can not be insisted upon too strongly. The majority of the points are familiar to most of us, but to the young physician particularly it is easy to point out discrepancies between actual facts and those laid down in the text-books. It is especially important in the early stages of phthisis, where the disease is localized in the beginning. There is a decided or a slight change from the normal if , the disease is limited to a small portion of the lung and that is a grave sign. We must remember that similar changes occur in bronchitis and, not infrequently, in pneumonia. This should be taken into careful consideration before pronouncing diagnosis. In com paring the two sides the statement that the back is better than the front does not correspond with my observations. I under stand perfectly well that we can get clearer sounds over the lower lobe of the lungs than elsewhere, but in comparing the two sides, in the majority of cases, you can get better results in front than posteriorly. You can count more upon the changes heard from in front. There is a disparity between the two sides in 50 per cent. of the cases. While the paper directs proper attention to the early signs, we should be especially careful that these early signs are not confounded with other signs which have nothing to do with tuberculosis. I see a large number of patients sent to me by other physicians where there has been an error in diagnosis in pronouncing the case one of tuberculosis. I have seen many lamentable cases where the patients have been told that they had tuberculosis and they were made miserable for life and driven to an early grave. I have in mind now two ladies, occupying high positions in society, who were condemned to die with tuberculosis ; in neither case was there any tuberculosis.

A sign not spoken of in the paper seems to me to be of greater importance every year, and that is the pulse. A rapid pulse of 115 to 120 in a person otherwise well points pretty positively to tuberculosis. Bronchial breathing is practically found in all patients suffering from pleurisy, over the eff usion, but not as continuously as over solid lung. In the text-books the voice sounds are represented as being present over the fluid. In not one out of fifty will you be able to get voice sounds over the fluid.

Dr. James Tyson of Philadelphia-Dr. Bridge struck the keynote in speaking of pleurisy with effusion in the fact of diminished fremitus, which always means pleurisy with effu sion. There is, however, one exception, which occurs in chil dren with a purulent effusion as contrasted with serous effusion; sometimes there is an increase of fremitus. This is said to be the case, although I have never realized that such was so

Dr. C. E. Enson of Denver-I want to add to the remark made by Dr. Tyson in regard to the rapidity of the pulse not only occurring in advanced cases, but in the incipient ones; the rapidity of the pulse is a sign which has made me very suspicious in many cases. It is not only the rapid pulse which makes me suspicious, but also the irritable pulse. It is the nervous irritable pulse of small caliber that makes me sus picious of the early stages. This is not only a diagnostic sign, but one of certain prognostic importance, particularly in cases in this high altitude. It is a pulse which does not slow down in a reasonable space of time. If the pulse continues to be irritable the cases do not do well in the long run.

Dr. BEGGS, of Denver-The paper is an extremely important one, especially to the younger members of our profession. remember one statement made ten years ago by Dr. Glascow, of St. Louis, Mo., which has always impressed me; it was that no man was capable of diagnosing incipient phthisis until he had examined one thousand chest cases. What he meant was, the diagnosis of the early cases. This diagnosis depends upon the alterations in the quality, and this is extremely hard for students-yes, medical men, too-to learn to recognize in the early stages. I have found that statement to be true to a certain extent in my own experience. I am not sure of my own ability to make an early diagnosis, although I have been practicing thirteen years. In cases of incipient tuberculosis there is an inconstancy in the occurrence of vocal fremitus. I have found many cases which have prastically the same anatomic conditions present, in which the character of the vocal fremitus and resonance varies; and, even in the same patient this varies from time to time just sufficient to make vocal resonance a matter of doubt. I also find that I must agree that the front of the chest is better for comparisons than the back.
As regards pleurisy, $I$ have in mind a case of a child 14 years of age, in whom the right pleural cavity was nearly filled with a serous effusion, as was proven by puncture; in this case there was no bulging, and the measurement of the chest showed no difference between the two sides. And, so far as we could detect, there was no difference in volume in the two sides of the chest.

There is another sign which has given me more trouble than any other, which is mentioned in most text-books, and that is the muscularis sussurus; this has been to me a source of error in making diagnosis. This, too, is a sign that every student should have his attention called to.

Dr. BRIDGE-As 1 stated at the beginning of this paper, I intended to call attention to a few of the possibilities and like. lihood of error in the diagnosis of chest diseases, particularly on the beginning of such diseases as phthisis. I should have made mention of the errors which might occur on account of the muscular action; this is a source of error and great trouble to young practitioners in listening to chests, where particular care should be taken to place the muscular fibres of the chest wall at rest. Here muscle tones are produced by contraction which would interfere with intra thoracic tones if one be not careful.

It is possible that I did not make myself sufficiently well understood in speaking of comparing the two sides of the chest. In the beginning of phthisis at the apex, I have been better satisfied with an examination made from in front of the chest. Slight flbrosis begins above and extends downward, and frequently this fibrosis has so far extended as to interfere with the accuracy of this examination when made from in front. Then, too, you do not have confused condition of the heart sounds as in front. If you find that there is evidence of fibrosis extending below the fissure between the two lobes of the lung posteriorly, it is a very strong evidence that there is a lesion of considerable consequence existing above it. Another point I wish to emphasize is that the patient should be made to expire profoundly. I can mention many cases during the past two years where the diagnosis could not have been made of incipient phthisis without this fact being known.

In answer to Dr. Tyson I would simply say that I do not recall any instance where the tactile fremitus from the voice was greater over the accumulation of pus than over the opposite side.

I am amused at the statement made that one should examine one thousand cases of chest diseases before one is capable of distinguishing the slightest changes that occur in incipient phthisis. Practically that statement is true, though I do not believe that this is necessary.

\section{ANEURYSM OF THE CONCAVITY OF THE TRANSVERSE ARCH, APPEARING EX. TERNALLY AS A LARGE TUMOR IN THE REGION OF THE HEART.}

Presented to the Section on Practice of Medicine at the Forty.ninth Annual Meeting of the American Medical Association, beld at Ienver, Colo., June $7-10,1888$.

BY H. W. McLAUTHLIN, M.D., AND W. N. BEGGS, M.D. DENYER, COLO,

Charles M., aged 52 years, musician, entered the Arapahoe County Hospital, Denver, March 9, 1898. His mother died of cancer, at the age of 70 years, and a sister of his mother died of phthisis. $\mathrm{He}$ had been given to dissipation, contracting syphilis when about 20 years old, and he was addicted to drink. $\mathrm{He}$ had contracted gonorrhea several times. During the last fifteen years he had had some rheumatism. He said his present trouble commenced four months before, with a bad cold acccompanied by a severe cough, at which time a swelling appeared in the left chest, which had gradually increased.

The tumor had been tender and attended by shooting pains, moderate in character and confined chiefly to the region of the swelling. About two months before entering the hospital he became short of breath and this had increased so that he could with difficulty move about. He had coughed considerably and expectorated freely. His appetite had continued good but he was inclined to diarrhea. He had lost about twenty pounds in weight during the four months. He had also had some diffculty in swallowing.

Physical examination.-Patient is of small stature and stoops. He appears apathetic and weak. His memory poor. He answers the same questions differently at different times. Scars, apparently syphilitic, are abundant on legs and back. A tumor, the size of two large fists, on the left side of the sternum, extends from the lower border of the second rib to 
the upper border of the seventh rib, and laterally from the center of the sternum to the middle of the axillary region. The tumor is firm to touch, but pulsates distinctly under the fingers, more especially at the base. No distinct bruit is audible. The position of the heart can not be determined, although the sounds are distinctly audible in the interacapular region and throughout the right side. In the left axillary region, just below the tumor, a systolic murmur is audible; flatness existe over the whole chest. The respiratory sounds are feeble and fremitus is absent, except in the upper part near the spine, where the breathing is bronchial with coarse râles. The right side shows exaggerated respiration in spots, with moist rales, the last being more abundant in the lower back. There is some edema about the ankles and the eyelids show distinct puffiness. The pulse averaged about 90 per minute; respiration, 39 . In the right radial artery pulsation is scarcely perceptible, in the left it is present but extremely weak, Pulsation in the carotids is apparently equal. There is no tracheal tugging. The right pupil is larger than the left, the latter being smaller than normal, accompanied by some ptosis. But convergent strabismus in the right eye, the patient claims, is of much longer standing than the tumor. Slight hoarseness is present. Urine analysis negative.

He became progressively weaker. His temperature varied from 96 to 100 , being below normal more than half the time. The tumor continued to enlarge perceptibly in every direction. On March 26 it is noted as extending from the clavicle to within four inches of the lowest rib, and laterally from the posterior part of the left axillary space to a point two inches beyond the right border of the sternum. The surface meas uremente were, vertical ten inches, transverse thirteen inches.

Dr. E. J. Rogers, the attending surgeon, saw the patient in consultation soon after his admission. A number of other physicians, especially Drs. Eskridge, Whitney and Sewall, made careful examinations. Dr. Rogers, by the exploring needle, withdrew a small amount of straw-colored liquid from the left pleural cavity. It was not, however, deemed wise to withdraw a considerable amount of fluid, although doubtlese present, as it would tend to interfere with the established resistance hitherto offered to the advance of the tumor in certain directions. About ten days before death a reddened appearance of the skin at the summit of the tumor was noticed, as if the skin were about to ulcerate. A day or two later blood began to appear at this place, the most of it clotting as it appeared on the surface. It continuously stained his clothes. On several occasions he had requested the loan of a knife from neighboring patients, and he was put under more careful watching, as a mild delirium became manifest.

Early on the morning of March 28 he became quite suddenly and extremely distressed for breath. He complained of severe pain but could not locate it. He received temporary relief from one-quarter grain of morphin, hypodermically; about six hours afterward he began struggling for breath, tearing at his throat and was unable to speak. He conveyed the idea to those around him that his throat was closing up. There was apparently spasm of the larynx. Respiration ceased about thirty minutes later.

Postmortem examination by W. N. Beggs, M.D., four hours after death. It is the body of a middle-aged, rather emaciated, man. Rigor mortis is established and livories are present in the dependent (posterior) parts not subject to pressure. The eyes are dulled. Projecting from the left thorax is a large, almost hemispheric tumor. It extends from the left anterio axillary line to one inch to the right of the sternum and from the clavicle to the seventh rib. It is rather moderately firm to pressure. Over the anterior surface, including the area of the nipple, the skin is eroded and covered with dried, clotted blood for an irregular ovoidal space two by one and one-half inches in dimensions. In dissecting away the skin over the tumor it was found that as this eroded area was approached the skin became progressively thinner, the subcutaneous tissue disappearing entirely and the cutis vera becoming so thin that, although great care was used, it was impossible to avoid invading the tumor itself. This having been done a very considerable amount of cruor was expelled and it was found that the tumor was saccular, communicating with the aorta. The eroded distal extremities of the second and third ribs extended in to the cavity from the left and the fourth and fifth ribs were imbedded in the cavity, both being eroded, chiefly on the upper edges and under surfaces. The left edge of the sternum was eroded to a depth of about one-half inch, extending from the attachment of the second to the fourth ribs, the costal cartilages of which had entirely disappeared.

The pericardial cavity was entirely obliterated by adhesions of slight degree of firmness, not entirely recent, however. The heart, small, was turned to the right on its axis, the left ventricle turned toward the front. The heart muscle was pale, labby, rather friable, and of a uniform, diffuse, slightly yel lowish cast. Death had occurred in moderate diastole and all the cavities contained a slight amount of mixed blood clot. The valves were normal. The ascending and transverse portions of the arch of the aorta were considerably dilated and the inner surface was wrinkled and freely dotted with irregular, yellow, very slightly elevated patches (atheromatous). At the concavity of the arch, beginning one and one-eighth inches above the upper margin of the semilunar valves, was the opening of the aneurysmal sac. This was eight and one half inches in circumferance. The sac was directed first downward and forward, then forward and upward, compressing somewhat the left bronchus, although there were no traces of erosion therein. Externally to the bony thoracic wall, the wall of the aneurysmal sac seemed to be composed of the soft parts of the thoracic wall.

In the right pleural cavity there were numerous, firm, old adhesions throughout. The right lung presented no peculiarities except a slight amount of emphyeema. The left pleural cavity contained one and one-half quarts of a clear ambercolored fluid. The left pleura was much thickened and opaque. The left lung was very much compressed and consolidated, and contained no air, portions of it sinking in water. On cut surface it was of bluish-gray color, mottled with very many rounded and triangular-shaped patches, slightly elevated above the cut surface, varying in color from gray to yellow and in consistency from moderately firm to almost semi-fluid, the latter being characteristic of the yellow patches.

The liver was of approximately normal size. The upper surface was granular and covered with numerous shallow, richly. stellate scars. On the cut surface it was slightly granular with distinct lobular markings, mottled dark bluish-red and yellowish. The splenic capsule was much thickened and opaque, white in color. On the surface were a fow shallow stellate scars. Otherwise the spleen and the other organs not mentioned presented no peculiarities of special interest here.

A peculiarity of this aneurysm is the location of the external tumor, the usual place being beneath the sternum, below the right or left clavicle or in the back. A review of several authorities has failed to disclose so large an aneurysm, similarly situated. In case No. 150 in Hayden's work, the aneurysmal tumor extended from the level of the second to the eighth dorsal vertebra, occupying the position of the left scapula, which was displaced backward and outward. It was hemispheric and measured nine inches in the transverse and fourteen in the vertical diameter.

Differential diagnosis.-What were the signs and symptoms during life favoring the diagnosis of aneurysm and what other diseases should have been considered? The age of the patient, the history, the signs of syphilis, together with a pulsating tumor of the chest and pressure symptoms would certainly strongly suggest thoracic aneurysm. Yet the pulsation was nowhere strong, although toward the base a forcible and expansile pulsation was felt. Da Costa's most emphatic sign, viz., the presence of "two points of pulsation in the chest, two hearts apparently, each with its own distinct beat, its own distinct sounds," was absent, as the tumor included the ordinary cardiac area and the real location of the heart was not ascertainable. The absence of a thrill and of a murmur from the tumor is more corroborative than essential to the diagnosis, according to most authors, the number and solidity of clots in the tumor causing the variations in the signs to be derived from auscultation.

A pulsating empyema is said by all authorities to occasionally simulate aneurysm, especially when a pulsating tumor is situated in the region of the heart. In the case reported, physical signs of fluid in the left chest were present, viz, flatness, with absence of respiratory sounds and lack of fremitus. The tumor in this case was, however, enormoúsly large for an empyema pointing externally. Moreover, the tumor was firmer and the pulsation more expansile than 
would be expected in empyema; neither was the pulsation disseminated over the chest; unmistakable pressure signs were also present. The needle showed the fluid in the chest to be non-purulent.

The following case of pulsating empyema is reported in Stokes' work on the "Heart and Aorta":

The heart was displaced far to the right. "Each stroke of the misplaced heart produced the most th extraordinary diastolic pulsation of the whole left side. The bed was shaken at each beat of the heart, and the patient's sleep interrupted by the vast and violent throbbing of the side; yet the force of the heart did not seem much augmented. There was no appearance of any external pointing of the contents of the sac."

Solid tumors of the chest.-Sarcoma, lymphomata and lymphadenomata are infrequent and tend to grow inward rather than outward. Cancerous growths are more frequent. If such a growth projects externally and pulsates, the symptoms and signs may simulate an aneurysm with rather firm clots. The expansile force is usually, however, much less distinct. The adjacent glands of the axilla and neck are often enlarged. The veins on the side of the thorax affected are more apt to be distended and especially, according to Hayden, varicose. The amount and character of pain is unreliable as a differential symptom. It should be noted in passing, however, that the degree of pain in this case was far less than would be expected in either an aneurysm or a solid tumor as large as the one under consideration. It is possible that he may have previously had more pain, but failed to recall the fact owing to the weak condition of his mind. It is more likely that pain was not prominent on account of the pressure of the tumor being outward rather than inward. The general health is usually poorer in cancer. In this oase the general health was poor, hut the other conditions could account for it. Osler and other excellent authorities speak highly of the diagnostic value, in aneurysm, of tracheal tugging, first mentioned by Oliver. On the other hand, Wood and Fitz state that Grimsdale considers the sign of little positive value, "since he found it in 16 per cent of a number of persons free from aneurysm and examined with reference to its presence."

Mediastinal abscess. - The size and location of the tumor, the character of pulsation, the degree of pressure symptoms and the character of the temperature would all tend against this as a probable diagnosis. Nevertheless, although there was a general expectation to find an aortic aneurysm at the postmortem, that expectation fell considerably short of being a conviction in the minds of those who had examined this patient the most carefully. The fluid in the pleural cavity, being clear, thin and non-fibrous, was probably due to pressure. Osler speaks of aneurysm from the lower or posterior wall of the arch compressing a bronchus, thereby producing bronchorrhea, gradual bronchiectasy and suppuration in the lunga process which by no means infrequently causes death in aneurysm, and a condition termed at the Montreal General Hospital "aneurysmal phthisis."

Pepper speaks of an irregular type of fever in such cases, as occurred in the case here reported, and that chills and sweats may occur, more strikingly showing a septic process.

Lebert, who analyzed a large number of cases, found that the average duration of life after the first distinct symptoms of thoracic aneurysm was from fifteen to eighteen months. A number of cases, however, are on record where life was prolonged for several years, and in a few for even ten years after an external tumor had appeared. In Hayden's analysis of sixty-eight cases, death occurred from rupture of the aneurysm in twenty-six instances. In only one of these twenty-six was the rupture external. Nine of the sixty-eight cases died from asphyxia. In the case reported here death would probably have occurred in a very short time from external rupture.

In reviewing this subject one is struck with the faot that hemorrhage from aortic aneurysm is far from being always fatal. Slow oozing or even quite severe and repeated hemorrhages, if there are intervals in which to recover from the immediate shock, may extend over a considerable time. Osler reports a patient who had "several brisk hemorrhages and who died four years after, having in the mean while enjoyed average health." Where there is an external tumor signs are apparent before rupture occurs. Stokes relates the following case, in which there was an external tumor in the lower sternal region which had caused absorption of the bone. There had been discoloration of the skin, followed by signs of sloughing, "so as to display what seemed to be the outer surface of the coagulum of the aneurysm. At each systole of the heart this coagulum moved outward, so as to close up the orifice in the skin and then receded during the diastole. After some days the coagulum gave way and a deluge of blood poured out. Immediate death would have followed but for the presence of mind of the nurse, who, on the instant, snatched up a cotton apron and stuffed a portion of it into the opening of the chest." The patient lived many days in good health and spirits, his life, so Stokes says, "depending on the precarious support of a plug of cotton rag which at every stroke of the heart appeared on the point of being forced out of its situation. The result was of course fatal."

Osler says that spontaneous oure is not very infrequent in small saccalated aneurysms of the asoending aorta. In many of these cases, the disease has been unsuspected during life and the obliterated sac found accidentally at the postmortem. Wood and Fitz state that aneurysms of the arch of" the aorta "are always a source of danger and usually prove fatal, although they may exist for years. Their progress may be checked for a long time by the formation of thrombi, but the process of repair is always incomplete, and it is doubtful if any true aneurysm of the arch large enough to produce symptoms has ever been healed.'

Abut ten years ago a patient died at the County Hospital here whose physical examination gave dulness over a portion of the chest, which, together with other signs which have escaped my memory, could not be satisfactorily explained. At the autopsy we were surprised to find the remains of thoracic aneurysm, the laminæ of organized fibrin showing beautifully.

\section{THE PATHOLOGIC ANALOGY OF BRIGHT'S} DISEASE AND SYPHILIS.

$$
\text { BY W. H. WHITEHEAD, M.D. }
$$

atLANTA, Ga.

First having my attention called to the efficacy of specific treatment in a case of albuminurie, in which I gave the mixed treatment of potassium iodid an 1 